\title{
Revisión sistemática sobre síndrome de Burnout en personal de salud en América Latina entre 2015-2020
}

\author{
Systematic review on Burnout syndrome in healthcare personnel in Latin \\ America between 2015-2020
}
Calixto Tapullima Mori ${ }^{1}$, Eveling Nathaly Munguía Girón ${ }^{2}$, Elián Moisés Reyes Cántaro ${ }^{3}$, Erick Brandon Sánchez Gonzales ${ }^{4}$ Universidad Cesar Vallejo

Recibido: $07-01-21$

Aceptado: $03-05-21$

Publicado: $18-06-21$

\section{Resumen}

El estudio tuvo como objetivo evaluar el síndrome de burnout en personal de salud asistencial en bases de datos que contengan literatura científica en América Latina entre 2015 - 2020, se utilizó un enfoque cualitativo, básico, de diseño teórico de revisión sistemática, se analizó 43 artículos; para la búsqueda se revisó 5 bases de datos Scopus, Ebsco, Scielo, Dialnet y Redalyc, las palabras clave fueron "Síndrome de burnout" médicos, "síndrome de burnout" "personal médico", "Burnout" médicos y "burnout" salud, obteniendo así de Scopus (31), EBSCO (992), Scielo (86) Redalyc (783) y Dialnet (110), llegando a concluir así que la mayoría de los artículos proceden de Brasil 14, seguido por Perú (8), la totalidad de los estudios corresponden a un tipo aplicado, de los cuales 41 son descriptivo trasversales, mientras que solo 2 son de diseño correlacional, la muestra fue en su totalidad personal médico llegando a 11693 sujetos, finalmente se evidencia una alta prevalencia del síndrome de burnout al igual que en sus componentes, partiendo desde $4.1 \%$ hasta $100 \%$ del personal médico evaluado.

Palabras clave: burnout; revisión sistemática; realización personal; agotamiento; despersonalización.

\section{Abstract}

The objective of the study was to evaluate the burnout syndrome in health care personnel in databases containing scientific literature in Latin America between 2015 - 2020, a qualitative,

1 Universidad César Vallejo, Tarapoto, Perú.

Autor para correspondencia: calixtotapullima01@gmail.com ORCID: https://orcid.org/0000-0001-8036-2199

2 Universidad César Vallejo, Lima, Perú.

E-mail: evelingmg19@gmail.com ORCID: https://orcid.org/0000-0002-4075-9869

3 Universidad César Vallejo, Lima, Perú.

E-mail: goomy.458@gmail.com ORCID: https://orcid.org/0000-0002-6938-2989

4 Universidad César Vallejo, Lima, Perú.

E-mail: erickbsg@outlook.com ORCID: https://orcid.org/0000-0001-5917-9816

(C) Los autores. Este artículo es publicado por la Revista de Investigación en Psicología de la Facultad de Psicología, Universidad Nacional Mayor de San Marcos. Este es un artículo de acceso abierto, distribuido bajo los términos de la licencia Creative Commons Atribución 4.0 Internacional (CC BY 4.0) [https://creativecommons.org/licenses/by/4.0/deed.es] que permite el uso, distribución y reproducción en cualquier medio, siempre que la obra original sea debidamente citada de su fuente original. 
basic approach was used, with a theoretical systematic review design, 43 articles were analyzed ; For the search, 5 databases Scopus, Ebsco, Scielo, Dialnet and Redalyc were reviewed, the keywords were "Burnout syndrome" doctors, "burnout syndrome" "medical personnel," Burnout "doctors and" burnout "health, obtaining as well as Scopus (31), EBSCO (992), Scielo (86) Redalyc (783) and Dialnet (110), thus concluding that most of the articles come from Brazil 14, followed by 8 from Peru, the totality of The studies correspond to an applied type, of which 41 are cross-sectional descriptive, while only 2 are of correlational design, the sample was entirely medical personnel reaching 11693 subjects, finally a high prevalence of burnout syndrome is evidenced as well than in its components, starting from $4.1 \%$ to $100 \%$ of the medical personnel evaluated.

Keywords: burnout syndrome; systemic review; personal fulfillment; exhaustion; depersonalization.

\section{INTRODUCCIÓN}

La atención en salud representa un derecho fundamental, razón por la cual los prestadores de servicio tienen que estar en constante exigencia; sin embargo, que sucede cuando llega a un límite de sus actividades. Para mayo del 2019 el término "agotamiento" se incluye en la Clasificación Internacional de Enfermedades (CIE-11, 2020) con carácter ocupacional definiéndolo de esta manera como un síndrome que resulta del estrés crónico en el centro laboral, no habiendo podido ser manejado de manera exitosa (Organización mundial de la Salud, [OMS], 2019). La sobrecarga laboral en los colaboradores y la baja capacidad para lidiar con sus actividades pone en riesgo la salud física y mental de los profesionales de salud (Torrecillas et al., 2020; Saborio \& Hidalgo, 2015). Diversos estudios incluidos el de Muñoz et al. (2018) manifiestan que los individuos de interacción directa son más propensos a padecer de esta sintomatología, así el personal asistencial conforma un grupo altamente vulnerable.

Diversos estudios muestran que el personal médico aparece como grupo vulnerable para la prevalencia del burnout, lo que implica una necesidad de análisis (Torrecillas et al., 2020); de igual manera Vinueza et al. (2020) manifiesta que más del $90 \%$ del personal médico y enfermería de Ecuador presentan un moderadosevero síndrome de burnout asociados a su género, edad y la situación. Efectuando un comparativo con lo presentado por Ramírez (2017) quien evidencia solo un $4.2 \%$ de prevalencia de síndrome de burnout, la realidad actual demuestra que el agotamiento por la sobrecarga de trabajo en atención de salud juega un papel en la propagación de este síndrome, en ese sentido países como Colombia, Argentina, Chile y Brasil no presentan una realidad distinta. Por su parte Minsalud (2015) indica que los trabajadores médicos colombianos, suelen exponerse a múltiples riesgos en su entorno laboral los mismos que son reacios a la búsqueda de ayuda, lo que hace importante el abordaje de revisión. Su importancia en ese sentido, como lo demuestra García-Morán (2016) radica en que el padecimiento grave no 
solo tiene afección en los médicos que lo padecen, sino además se transfiere al usuario que viene siendo atendido.

Si bien dentro de un contexto peruano la presencia de ésta sintomatología "agotamiento profesional" está presente hace mucho tiempo, tal como lo menciona el Ministerio de la Mujer y Poblaciones Vulnerables (2017) afirmando que los operadores de salud se encuentran en un alto riesgo de padecimiento, estos están constantemente sometidos a contacto con el sufrimiento humano de manera muy reiterada, cotidiana y a tal punto crónico, recalcando además que el síndrome de burnout es consecuencia de eventos estresantes en el contexto laboral, es decir en este escenario con el rol de atención. Estos datos se afirman con anterioridad según Maticorena-Quevedo et al. (2016) quienes afirmaron que la prevalencia del Síndrome de burnout está presente hasta en el $66 \%$ de profesionales de salud peruanos, Solís-Cóndor et al. (2017) también manifiestan que el 18.7\% del personal tienen un alto riesgo de despersonalización, $10 \%$ cansancio emocional y $32.1 \%$ de baja realización personal, el mismo que presenta una afección muy significativa debido al rol que estos tienen en la sociedad. Finalmente haciendo un análisis de la realidad a nivel mundial, latinoamericano y nacional hoy más que nunca la prevalencia de Síndrome de burnout está en aumento, pues las labores se han sobrecargado para el personal médico asistencial, en la misma línea se han orientado el desarrollo de diversos mecanismos para apalear esta problemática tal como lo demuestra el Ministerio de Salud [Minsa] (2020) donde se establecen políticas para el aseguramiento de la salud mental de los colaboradores. Este escenario, demuestra que la prevalencia del SB ha estado latente desde hace mucho tiempo, razón por la cual un estudio de carácter sistémico podría contribuir en gran medida para conocer la presencia de estudios en América Latina. Se busca sintetizar conocimientos correspondientes a la prevalencia de síndrome de burnout en personal médico, enfermería, atención en primer nivel, etc., siendo los datos correspondientes a América Latina, esto ha sido impulsado por la realidad actual que viene suscitando, donde la constante presión al personal médico se considera que es más significativa, por otro lado está la búsqueda de información concerniente a las características y como estos se han venido presentando en las diversas revistas latinoamericanas. De acuerdo con la realidad presentada se plantea como problema ¿Cuáles son los principales hallazgos de síndrome de burnout en personal de salud asistencial, a partir de la literatura científica a nivel latinoamericana entre 2015 -2020? En tanto el objetivo fue evaluar el síndrome de burnout en personal de salud asistencial en bases de datos que contengan literatura científica en América Latina entre 2015 - 2020.

En el proceso de revisión se ha obtenido diversos estudios como lo presentado por Álvarez, et al. (2019) que tuvo como objetivo realizar una revisión sistemáticaexploratoria del síndrome de Burnout en médicos durante los últimos siete años (2012-2018) en Iberoamérica. El estudio fue una revisión sistémica con palabras 
clave para la búsqueda como "médicos, síndrome de burnout" obteniendo 52 artículos que luego del proceso de selección resultaron 23 que cumplían todos los criterios establecidos. Asimismo, las bases donde se extrajeron los artículos fueron Redalyc, Scielo, Scopus, ScienceDirect, Medlinem EBSCO, entre las principales conclusiones se han encontrado la existencia de una prevalencia elevada del agotamiento emocional conjuntamente con la despersonalización (45.88) además una baja prevalencia en realización personal de los colaboradores médicos, siendo esto en mayor prevalencia del género masculino íntegramente correlacionadas con variables más significativas como en tiempo de residencia (2-3 años), la procedencia, entre otros, también se logró evidenciar la vulnerabilidad a la que están expuestas los médicos para el padecimiento de síndrome de burnout. Loya-Murguía et al. (2018) tuvo como objetivo examinar trabajos realizados en Latinoamérica que establecen la frecuencia del síndrome de agotamiento (SA) en el personal de salud en países latinoamericanos, al igual que la observación de diferencias en prevalencia entre las especialidades médicas y compararlos con resultados de otras partes del mundo. El estudio fue una revisión sistémica, para la búsqueda de información se utilizaron palabras clave como "Burnout Syndrom and Latinoamérica and México", obteniendo así 119 artículos, reduciendo a 29 artículos que cumplieron con los criterios. Las bases de datos donde se extrajeron los artículos fueron de PubMed, las principales conclusiones encontraron que el SA tiene una frecuencia que oscila entre $2.1 \%$ y $76 \%$, las mismas que se encuentran dentro del rango a nivel mundial, sin embargo, dentro del contexto latinoamericano las horas tiene una sobrecarga en el campo hospitalario y atención primaria, además de la no existencia de medidas preventivas de SA, siendo estos factores que contribuyen en su aumento. Toala (2019) tuvo como objetivo determinar la prevalencia del síndrome de burnout en los hospitales de habla hispana, el estudio fue una revisión sistemática, a partir de un metaanálisis de artículos usó palabras clave como "agotamiento en residentes, burnout, desgaste profesional, médicos residentes, síndrome de burnout", obteniendo 1950 publicaciones en la primera búsqueda y luego de los respectivos criterios fueron seleccionados 30 artículos. Las bases de datos donde se extrajeron fueron Scielo, Medline, Redalyc, Pubmed y Journal. Es así que entre las principales conclusiones fueron que se trabajó con una muestra de 2269 colaboradores de salud residentes (médicos, enfermeros, otros) con 29 años en edad media, en ese escenario se evidenció que la prevalencia del SB es mayor en solteros, en mujeres más que en casados varones, alcanzando además un promedio de $57 \%$, en cuanto a sus dimensiones, agotamiento $43 \%$, despersonalización $35.8 \%$ y realización personal con un índice de $41 \%$, finalmente estos elementos que poseen una afección al desarrollo de las actividades tienen incidencia alta en la salud mental de los médicos residentes.

Díaz y Gómez (2016) tuvieron como objetivo la creación de un panorama sobre la investigación en burnout en América Latina. El estudio fue una revisión sistemática haciendo uso de palabras clave como síndrome de burnout, "síndrome de 
quemarse en el trabajo" y el nombre de los países latinoamericanos, de esta manera se revisó 89 estudios en los diversos países bajo los criterios seleccionados. Las bases de datos de donde se extrajeron fueron Science Direct, Scopus, Ebsco, Redalyc, Scielo y Google Académico, en ese sentido el estudio presentó como conclusiones que la prevalencia del uso de instrumento y conceptualización son los planteados por Maslach y Jackson, además de que los estudios son en su mayoría descriptivos correlaciones, finalmente, se concluye que la mayoría de los estudios hacen uso de teorías y métodos elaborados en países desarrollados sin el análisis del contexto situacional que se vive en los centros laborales de América Latina. Foronda et al. (2018) plantearon como objetivo la compilación de artículos que describen las características del síndrome de burnout en el personal de salud. El estudio fue una revisión documental, para la búsqueda de los datos se plantearon como como palabras clave "burnout" "paramédicos", "quemarse en el trabajo" y "personal de la salud", analizando 22 estudios que contaron con los criterios establecidos por los investigadores. Se buscó información en diversos repositorios como los de la universidad Autónoma de México, la perteneciente a la Universidad de Chile y la Universidad de Buenos Aires, en la que se llegó a concluir de manera principal que los instrumentos de mayor utilización para la evaluación son el de Maslach Burnout Inventory, en última instancia a pesar que el SB representa un constructo reciente presentando dentro de la ciencia de salud, este no ha sido estudiado con mayor énfasis ni mucho menos se ha establecido la prevalencia en los colaboradores del área prehospitalaria. De Arco y Castillo (2020) plantearon como objetivo analizar las contribuciones de los trabajos de investigación publicados de 2018-2020. El estudio fue una revisión de literatura haciendo uso de palabras clave "burnout, agotamiento emocional, síndrome del trabajador quemado, work stress, condiciones mentales", obteniendo de esta manera 60 referencias en la primera búsqueda, luego del análisis y establecimiento de criterios se seleccionaron 32. Las bases donde se efectuaron las búsquedas fueron Scopus, Ebsco, Dialnet, ScienceDirect, Scielo y el propio buscador de Google. De esta manera las principales conclusiones fueron, el estudio del síndrome de burnout no es exclusividad del personal médico o de salud sino también lo pueden llegar a padecer todo personal que enfrentar diariamente situaciones de estrés crónico, finalmente, el SB siempre ha sido y seguirá siendo el tema de estudio de diversos campos.

El síndrome de burnout fue abordado hace más de 40 años, Silva (2001) hizo mención del psiquiatra Herbert Freudenberger como el primero en asignarle una definición o carácter clínico en 1974, centrándose en el deterioro de la atención profesional y los servicios que este presta, en la actualidad la definición ha venido siendo mejor afinada. Serrano et al. (2017) indican que representa una crisis de autoeficacia que ocurre a nivel individual que está caracterizada por una gama de sensaciones, actitudes, expectativas y sobre todo experiencias negativas que da a lugar problemas de disconfort, de igual manera Carballo et al. (2014) y Martínez 
(2019) mencionaron que el burnout es un problema que repercute de manera significativa en la salud mental y física de los profesionales, teniendo una afección en la calidad de servicio asistencial. Si bien el análisis de las diversas teorías pueden asemejar al burnout con otra sintomatología, como el estrés crónico (comúnmente confundido), dentro del contexto laboral estos dos conceptos "burnout" y "estrés" son distintos fenómenos, "el primero hace referencia a procesos caracterizados por la temporalidad breve, mientras que el burnout es la respuesta o consecuencia de estrés laboral crónico muy consecutivo (Muñoz, et al., 2018), entonces se podrá afirmar que el burnout representa un resultado de la cronicidad del estrés constante del individuo (Vidotti et al., 2019).

Para el estudio se ha considerado diversos modelos teóricos que abordan el burnout, iniciando por el Modelo de Edelwich \& Brodsky (1980), quienes afirman que representa un proceso de desencanto hacia las actividades laborales, en las que se plantean las fases como: entusiasmo, esta naturalmente caracterizada cuando se toma un trabajo nuevo donde se resaltan las aspiraciones y alta energía que posee el colaborador, es decir en esta etapa la percepción de los problemas son pasajeras y solucionables. El modelo de Cherniss (1980) afirmó que el burnout es un proceso transaccional del individuo quien manifiesta estrés y tensión en el centro laboral, en la que se evidencian tres momentos, el desequilibrio entre las demandas laborales y el recurso individual disponible, la respuesta emocional a corto plazo que es a consecuencia del desequilibrio anterior, desencadenando ansiedad, tensión, agotamiento, fatiga, entre otros, finalmente se aprecia el cambio de actitudes y conductas. El modelo de Price \& Murphy (1984) mencionaron que el síndrome se asemeja a un proceso de duelo, donde el individuo pasa por tres niveles, el personal, interpersonal e intrapersonal, es así como plantean seis fases sintomáticas como la desorientación, inestabilidad emocional, culpa, soledad, alivio y restablecimiento.

Modelo tridimensional, este síndrome comprende una respuesta del individuo al estrés laboral crónico, estas suelen estar conformadas por actitudes, negatividad de sentimientos para con las personas con quien trabaja o por el mismo rol que desarrolla; por otro lado este síndrome se caracteriza por un sentimiento de agotamiento, en ese sentido se considera una variable tripartido con características definidas en el individuo emocionalmente agotado, la despersonalización al igual que la baja sensación de realización personal en el centro laboral el síndrome de burnout es una respuesta al estrés laboral crónico, conformado por actitudes y sentimientos negativos hacia las personas con las que se trabaja, y hacia el propio rol profesional, siendo un síndrome caracterizado por la vivencia de encontrarse emocionalmente agotado, así también se configura como un síndrome tridimensional que tiene como característica, el agotamiento emocional, despersonalización y reducida realización personal (Maslach et al., 2001). 


\section{MÉTODO}

\section{Enfoque, tipo y diseño}

Se utilizó un enfoque cualitativo, donde se efectuó un análisis de casos (Sánchez, 2019), es decir, recaba datos que no tienen por finalidad la respuesta o prueba de hipótesis (Del Cid et al., 2007). El estudio se consideró básico, debido a que se efectuó un análisis de las teorías y estudios; además, fueron conformados por todos los trabajos de revisión efectuados que no son el informe de una investigación empírica (Montero \& León, 2002); es decir los estudios originales primarios (Ramírez et al., 2013). En el análisis de la literatura se presentaron un diseño teórico, de revisión sistemática (Ato et al., 2013), es decir los datos son recuperados, analizados e interpretados en el momento actual (Ato \& Vallejo, 2015).

\section{Participantes}

Es importante recalcar que los autores Bologna (2013) y Hernández et al. (2014) mencionaron que la población es todo conjunto de unidades que representan un estudio en particular, estos pueden ser individuos, animales, cosas u otros objetos que tienen en común ciertas características en relación con un tiempo y espacio determinado. Es así como la población concerniente a esta investigación de revisión sistémica son los diversos artículos sobre el síndrome de burnout en personal de salud asistencial en América Latina correspondientes al periodo 2015-2020. Bologna (2013) mencionó que la muestra es una parte representativa de la población que se ha identificado previamente; naturalmente permite que se llegue a una conclusión general a partir de la muestra estudiada (Supo, 2014). De esta manera poder efectuar la respectiva medición o estudio de la variable (Otzen y Manterola, 2017). Entonces para la revisión se analizó 43 artículos, se utilizó un muestreo tipo no probabilístico intencional (Otzen y Manterola, 2017), es decir para el estudio se seleccionaron artículos específicamente sobre síndrome de burnout en personal de salud asistencial en América Latina del 2015-2020.

\section{Procedimientos}

Para la búsqueda de los artículos se revisó las bases de datos como Scopus, Ebsco, Scielo, Dialnet y Redalyc, se utilizaron como palabras clave: "Síndrome de burnout" médicos, "síndrome de burnout" "personal médico, "Burnout" médicos y "burnout" salud. En el estudio se consideró solo artículo comprendidos entre los 2015-2020, de igual manera se aplicaron los criterios de inclusión y exclusión, eliminando los artículos duplicados, de esta manera se alcanzó la muestra de trabajo. Luego de la obtención de información se procedió a la revisión de cada uno de los artículos para la elaboración de una tabla de resultados sistematizado para la interpretación de resultados y discusión. 


\section{Instrumentos}

La técnica utilizada fue el análisis documental (Ñaupas et al., 2018) manifiestan que es el investigador quien accede a la información previa para establecer un conocimiento significativo sobre el síndrome de burnout en personal de salud asistencial, en ese sentido se utilizó como instrumento una matriz o tabla de registro de acuerdo a la base de datos desarrollado por los autores según la necesidad del estudio, la tabla sistematiza elementos principales como el año, autor, país, objetivo, muestra, conclusiones y hallazgos importantes.

\section{Consideraciones éticas}

Este trabajo de investigación respeta la ética y no apropiación indebida de materiales extranjeros sin citación o mención, por lo que se respetan los derechos de autor. De igual manera, al hacer uso de otros trabajos académicos fueron debidamente citados, además de haber sido estudiado y revisado la fuente de ubicación, finalmente la información no fue alterada ni parcial ni totalmente a convenientica del estudio.

\section{RESULTADOS}

Para el desarrollo del estudio se ha considerado necesario la explicación de la selección de información mediante el diagrama (Ver Figura 1).

Se describe el proceso seguido para la selección de los diversos artículos científicos consultados sobre el tema (síndrome de burnout en personal de salud asistencial en América Latina). Se observa que se efectuaron las indagaciones en cinco bases de datos, específicamente en Scopus $(n=31)$, EBSCO $(n=992)$, Scielo $(\mathrm{n}=86)$ Redalyc $(\mathrm{n}=783)$ y Dialnet $(\mathrm{n}=110)$ en el primer análisis de búsqueda con un total de 2002 resultados.

De igual manera, posterior a la revisión de los títulos y resúmenes se seleccionaron 107 artículos, éstos cumplieron con los criterios de inclusión, luego se dio paso a la revisión de los artículos completos, a los mismos que se aplicaron los parámetros de evaluación con respecto a la calidad, tal como se señala en la tabla 1, de esta manera se quedó con 83 artículos, en última instancia se procedió, luego de excluir a los artículos duplicados (40), considerar el estudio con 43 de los mismos, los mismos que fueron los incluidos para el desarrollo de la revisión respectivamente.

Se evidencia la sistematización de los artículos sobre el síndrome de burnout en personal de salud asistencial en América Latina comprendidas entre los años 2015-2020, de acuerdo con la revisión se han identificado que la gran mayoría de los artículos provienen de Brasil, siendo estos 14, seguido del mismo en frecuencia de artículos es Perú con un total de 8 . En consideración al tipo de investigación la totalidad de estos (43) son no experimentales, en estos estudios se han presentado diseños descriptivo-transversal (41) y correlacional (2). De acuerdo con el análisis de la muestra de la totalidad de artículos revisados (43) son el personal médico de 
centros hospitalarios, que trabajó con una muestra mínima de 18 residentes y 5062 médicos como máximo. De los objetivos evidenciados, los que más se repiten son los orientados a determinar la prevalencia del síndrome de Burnout en médicos, seguida de los mismos se ubica los objetivos de identificar la prevalencia de burnout en personal médico, todos estos con la intención de recabar información que revele el grado o nivel del síndrome presente en el personal médico. Un análisis de los instrumentos evidencia que el 95\% (41) han utilizado prevalentemente el Inventario de Burnout de Maslach, solo uno ha hecho uso del CBP-R y otro ha utilizado el Cuestionario de Evaluación del Síndrome de Quemarse por el Trabajo (CESQT) los mismos que han orientado al cumplimiento de las metas planteadas.

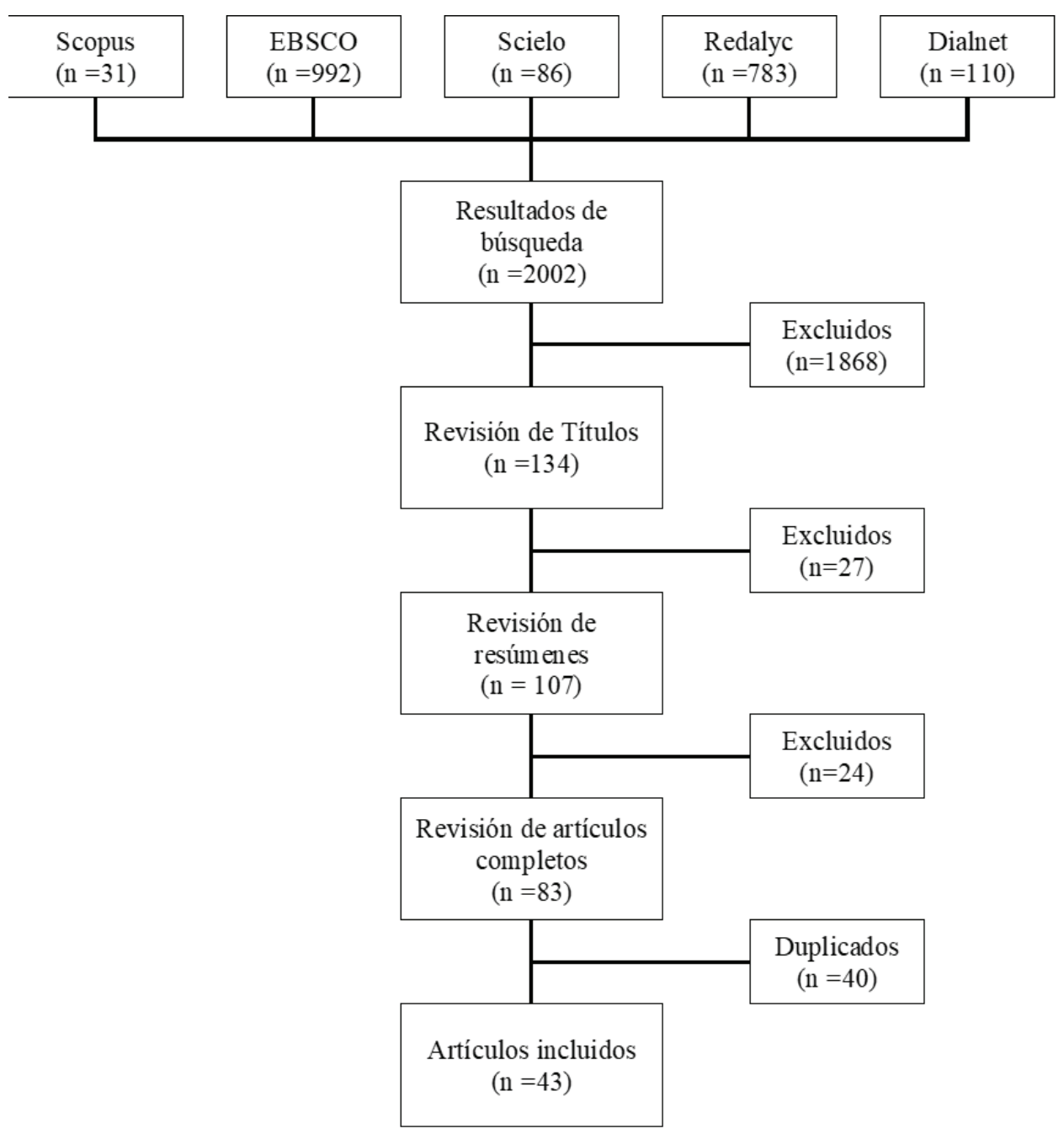

Figura 1. Diagrama de flujos de proceso de selección de artículos científicos 


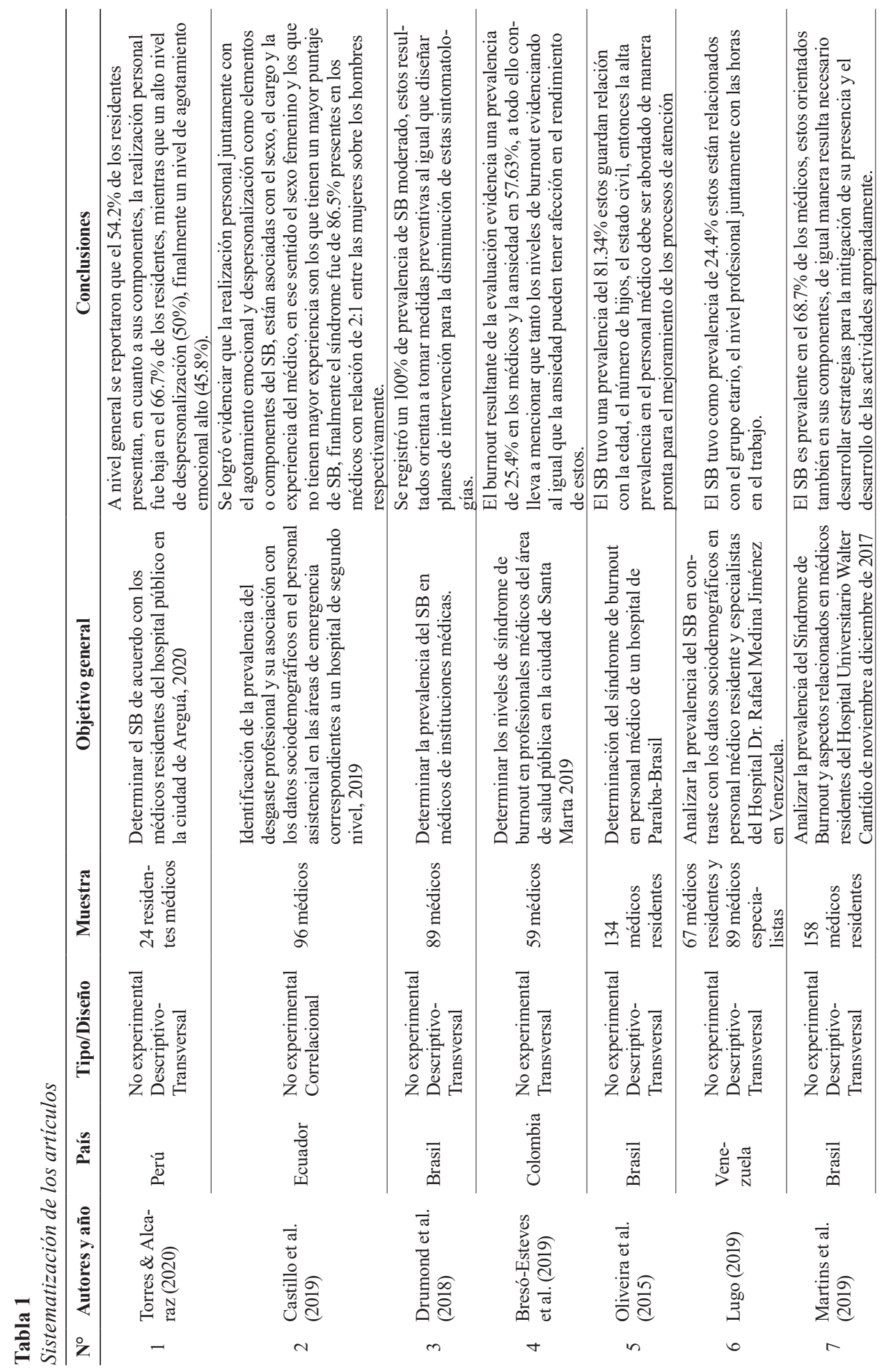




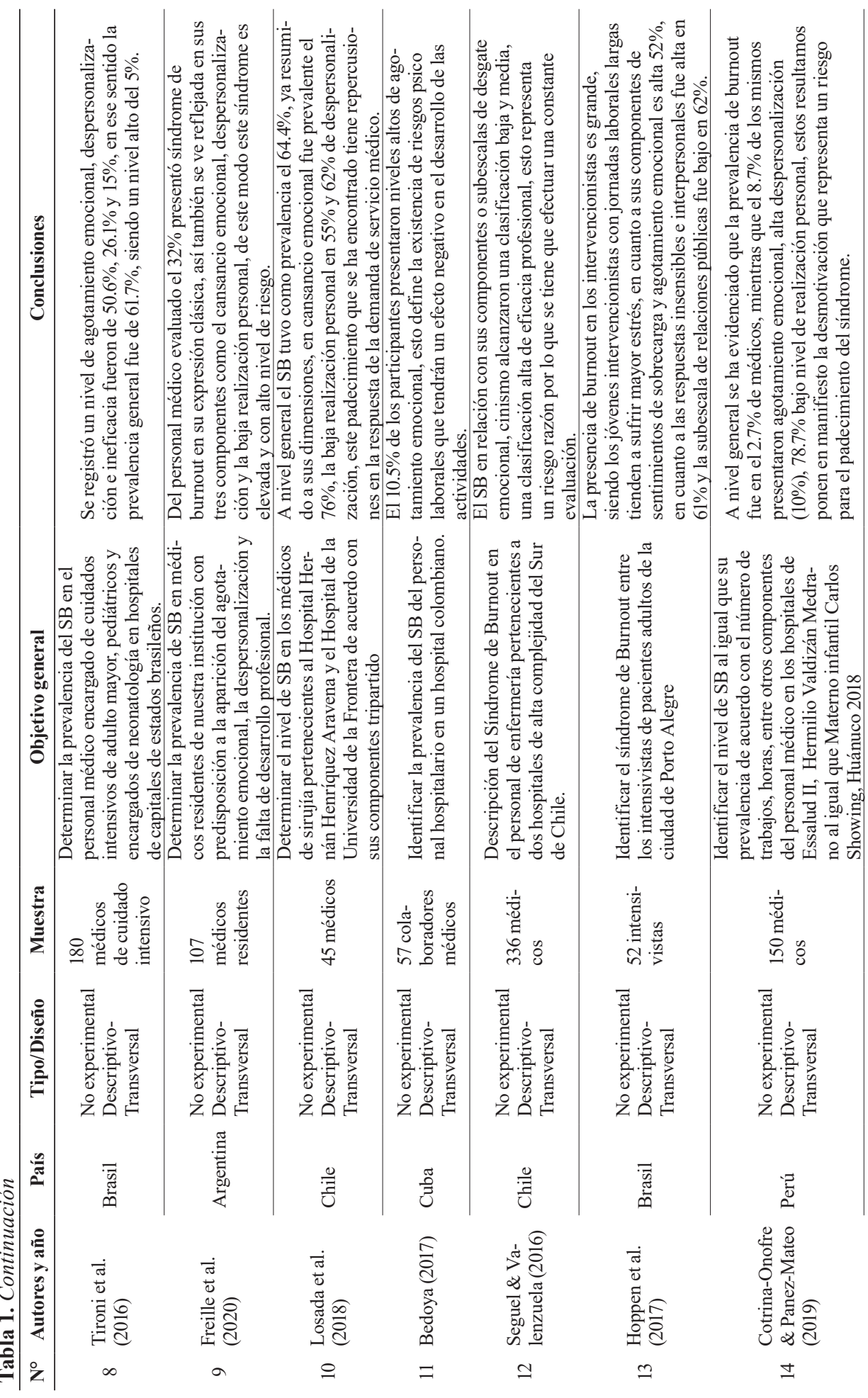




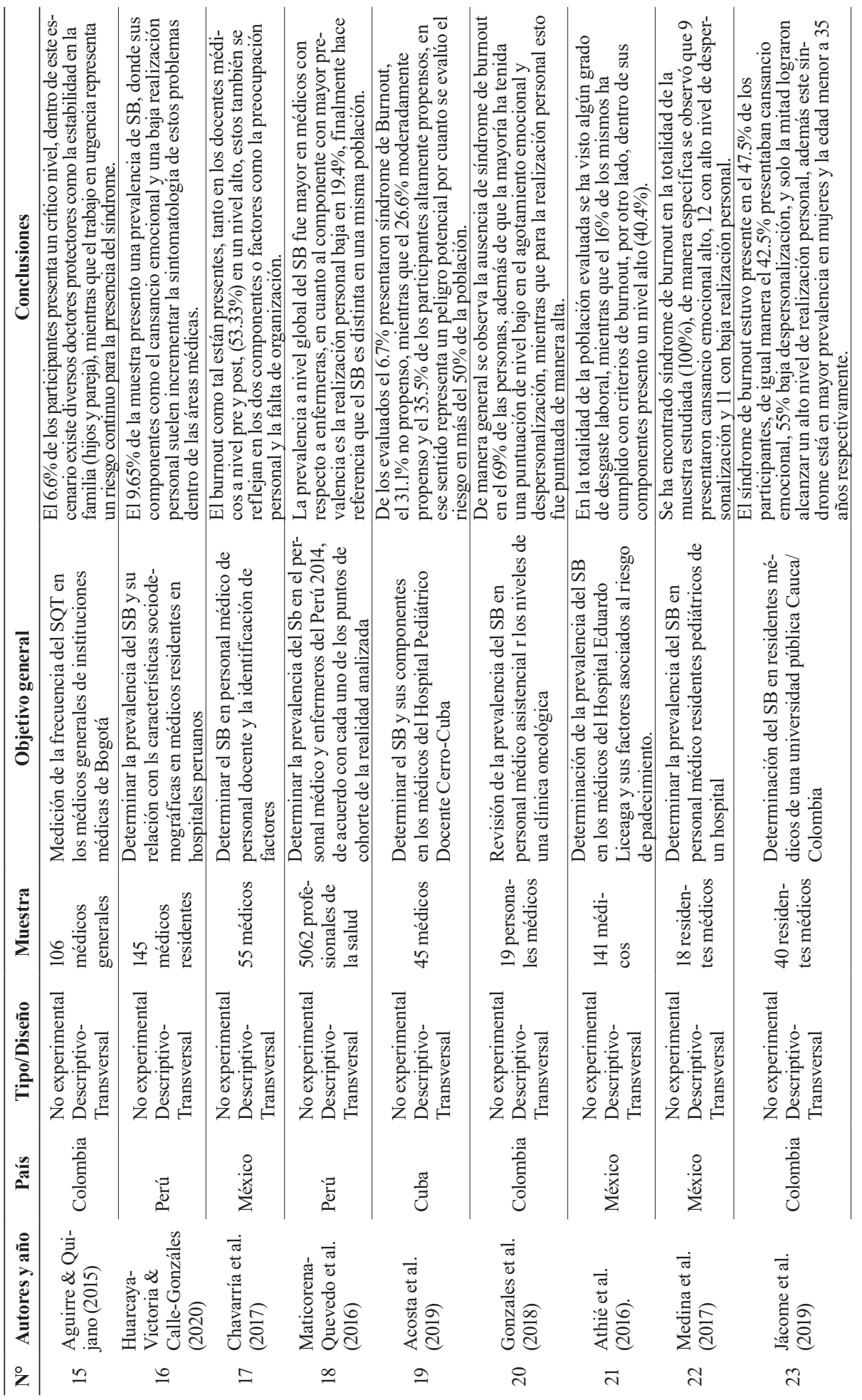




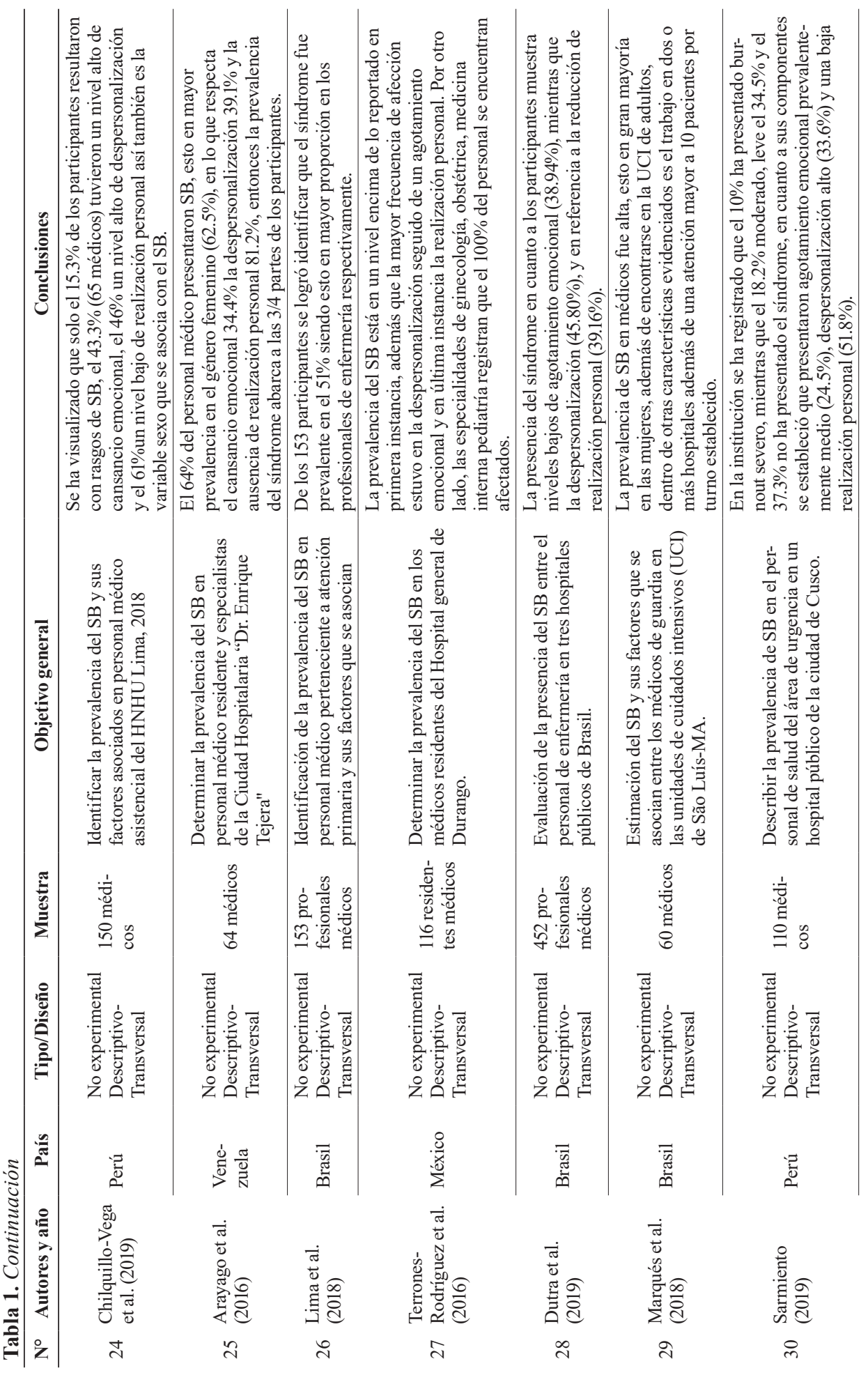




\begin{tabular}{|c|c|c|c|c|c|c|c|}
\hline & 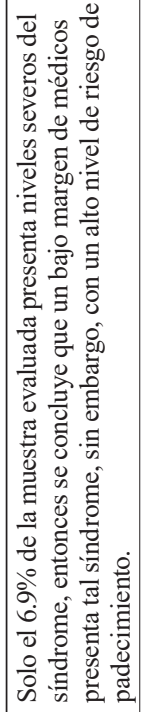 & 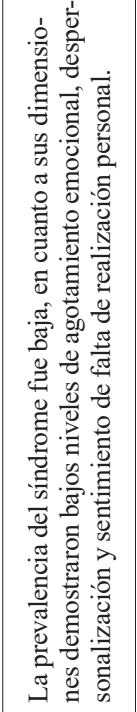 & 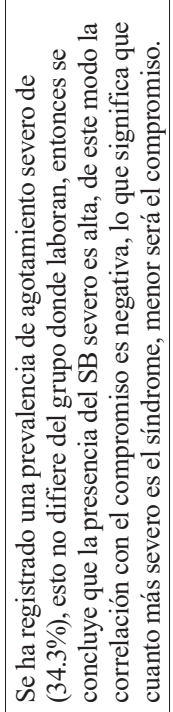 & 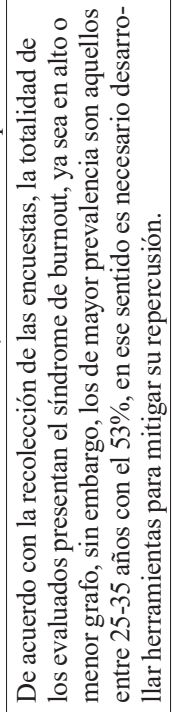 & 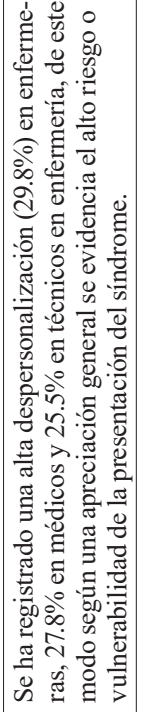 & 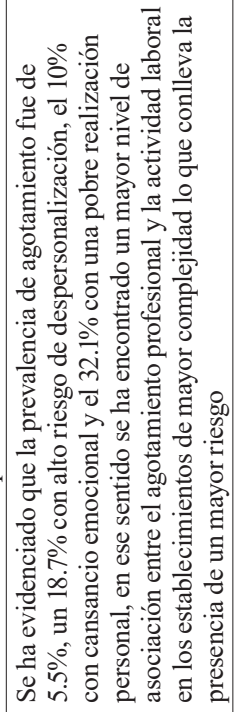 & 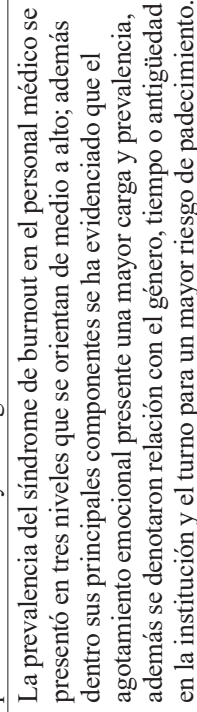 \\
\hline 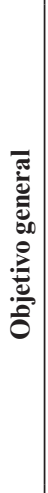 & 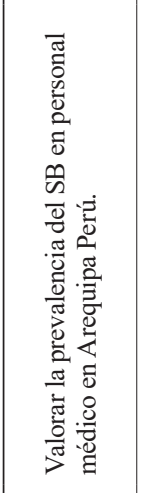 & 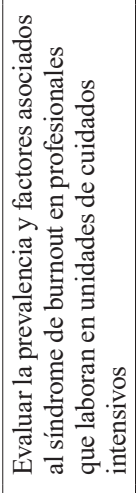 & 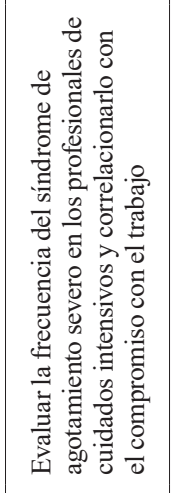 & 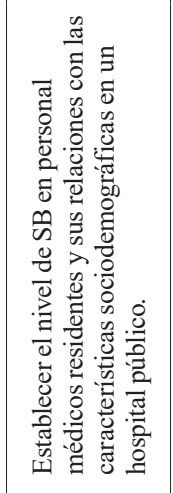 & 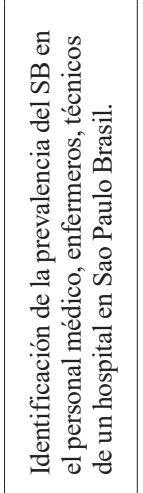 & 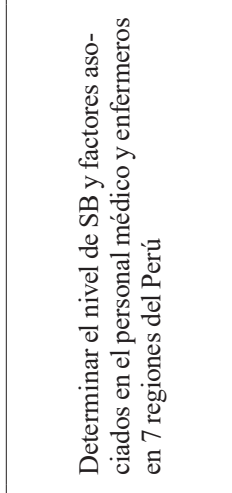 & 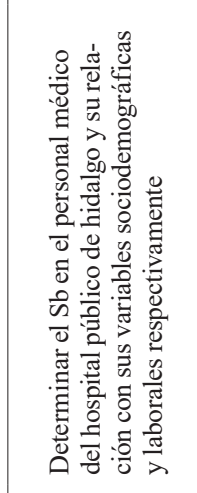 \\
\hline 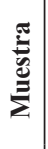 & 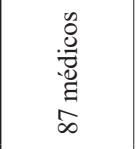 & 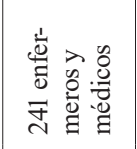 & 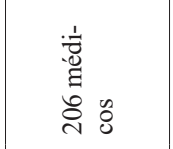 & 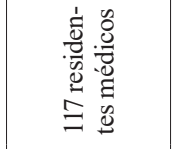 & 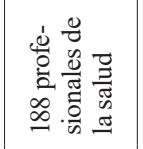 & 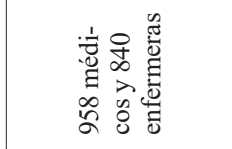 & 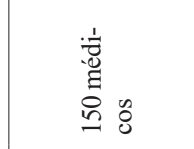 \\
\hline 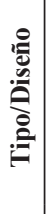 & 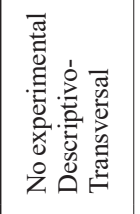 & 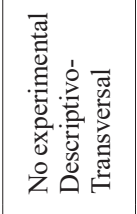 & 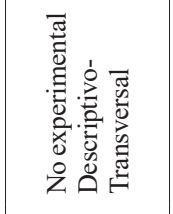 & 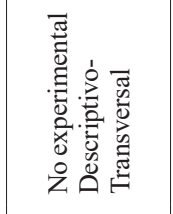 & 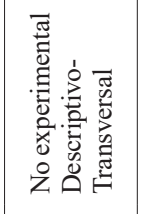 & 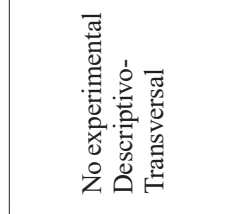 & 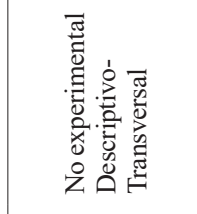 \\
\hline 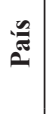 & 苛 & $\begin{array}{l}\overline{\vec{n}} \\
\overline{\tilde{n}} \\
\bar{n}\end{array}$ & $\begin{array}{l}\overline{\bar{n}} \\
\overline{\tilde{n}} \\
\bar{n}\end{array}$ & $\stackrel{\mathscr{Z}}{\tilde{\Xi}}$ & $\begin{array}{l}\overline{\bar{n}} \\
\bar{n} \\
\bar{n}\end{array}$ & $\begin{array}{l}\vec{D} \\
0 \\
0\end{array}$ & 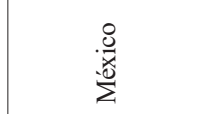 \\
\hline 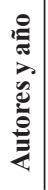 & 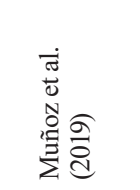 & 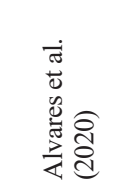 & 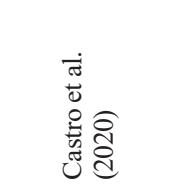 & 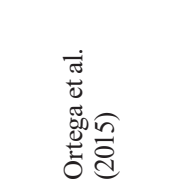 & 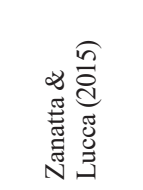 & 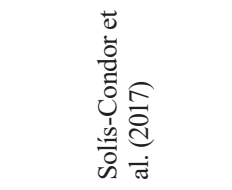 & 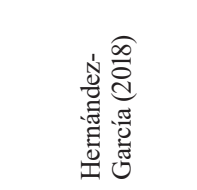 \\
\hline$\stackrel{\circ}{z}$ & $\bar{m}$ & లె & $\hat{m}$ & mे & $\ddot{m}$ & ల్ల & \\
\hline
\end{tabular}




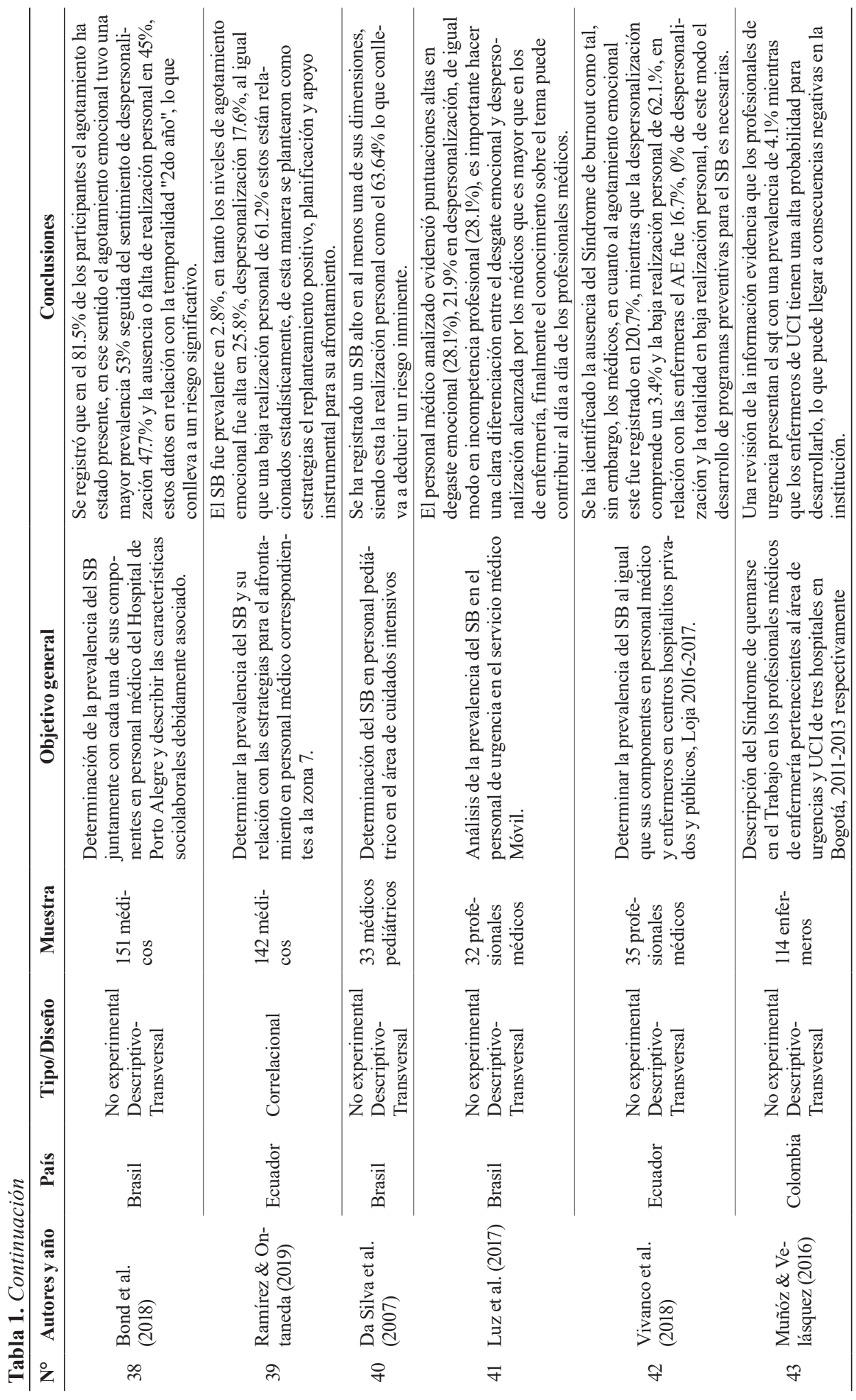


En relación a las principales conclusiones alcanzadas, se vio prudente la división de acuerdo al diseño de investigación efectuado, toda vez que tuvo un solo tipo (Aplicada); en ese sentido las conclusiones de los descriptivos-transversales registran niveles de SB desde $4.1 \%$ hasta el $100 \%$ de la muestra evaluada, donde además sus componentes tienen una prevalencia alta, sin embargo, de los estudios revisados solo 3 enfatizan la baja prevalencia o ausencia del mismo, en contraste con los estudios correlacionales se llegaron a establecer la relación con las variables o componentes identificados como el sexo y edad entre otros involucrados.

\section{DISCUSIÓN}

El estudio presentó como objetivo evaluar el síndrome de burnout en personal de salud asistencial en bases de datos que contengan literatura científica en América Latina entre 2015 - 2020, en ese sentido se detallaron los principales hallazgos obtenidos, de acuerdo a la sistematización de los resultados, en cuanto al análisis de procedencia de los artículos se ha observado que esta es predominante de Brasil (14 artículos) seguido de Perú con 8 artículos, en las que se observan una brecha significativa con los demás países latinos, guarda relación con lo planteado por Miguel (2011) quien manifiesta que la producción científica en América Latina se encuentra distribuida de manera desigual, esto también se evidencia en la distribución de artículos obtenidos por Díaz et al. (2016), donde efectúa una evaluación con estudios del síndrome de burnout que resulta una prevalencia mayor de países como Colombia, México y Brasil; sin embargo la muestra de estudio pese a tener como participantes a distintos trabajadores de la salud como el caso de médicos, enfermeras, auxiliares de enfermería entre otros, que guardan relación con el estudio actual planteado, también ha considerado otro grupo de profesionales, por cuanto estos tienden a atender de manera directa a un usuario, cliente, beneficiario como lo son los abogados, asistentes sociales, trabajadores sociales, familiares que se encargan del cuidado de enfermos, operadores telefónicos entre otros. En tanto el estudio presentado por Tabares-Díaz (2020) clasifica como primer lugar la producción brasilera con 31 artículos; es así que se considera que en los últimos años se ha presentado un mayor auge los cuales comprenden del 2012 al 2018 como manifiesta Mugnani (2018), así también menciona que fue debido a la necesidad de evaluación de salud mental solicitada en gran medida; de igual manera el estudio de burnout se efectúa a consecuencia de que los trastornos mentales como tal en las últimas décadas destacan un grave problema en el campo ocupacional y los servicios médicos (Baldonedo-Mosteiro et al., 2019).

El estudio también ha presentado en su totalidad un tipo aplicado, con el 94\% descriptivo transversal, los mismos que se caracterizan por la descripción de las variables, sus componentes e indicadores a fin de indagar la realidad de los elementos en el contexto estudiado (Rojas, 2015). Estos guardan relación con lo mencionado en su estudio Toala (2019) que de 30 artículos analizados 28 presentan 
diseños descriptivos, además Juárez-García et al. (2014) menciona en su estudio de revisión que el $93.75 \%$ presentaron diseños transversales, descriptivos, es así que resulta importante mencionar que en su mayoría se hacen uso de tipos cuantitativos debido a que permite una mayor interpretación o sistematización permitiendo así tener un mejor panorama de las variables estudiadas, además de evidenciar la aplicación de instrumentos que evidencia o refleje un panorama del ámbito de estudio (Urra \& Barria, 2010). Cabe recalcar que en el estudio el 95\% de los estudios han hecho uso del Inventario de Burnout de Maslach al igual que los postulados teóricos, el mismo que guarda relación con lo planteado por Díaz y Gómez (2016), quienes dentro de sus principales conclusiones afirman también la prevalencia del uso en cuanto al instrumento y conceptualización planteado por Maslach y Jackson, del mismo modo Foronda et al. (2018) concluye también que se han utilizado en mayor nivel para la evaluación el inventario planteado por Maslach, en tanto se suele hacer uso del instrumento por cuanto su constructo es el más acertado para la evaluación delo mismo, tal como menciona el modelo tripartido, donde se hace énfasis en el agotamiento emocional, despersonalización y la realización personal, los mismos que están presentes en lugares o trabajos con las que la atención de un individuo sobre otro es constante (Maslach et al., 2001).

Los estudios que se han revisado en su mayoría presentan objetivos descriptivos $(95 \%)$ como lo son la búsqueda de prevalencia de síndrome en médicos, seguida del objetivo identificar la prevalencia del burnout, si bien estos han contribuido de manera significativa en el estudio, también es necesario hacer mención acerca de los 2 artículos de diseños correlacionales, los mismos que lograron establecer su relación entre variables de sexo, tiempo de trabajo y edad, es así que guarda relación con lo obtenido por De Arco y Castillo (2020) quienes también efectúan objetivos para identificar, medir o calcular la presencia del síndrome de burnout en personal médico, sin embargo, se tiene que tener en claro que no es una exclusividad del personal hospitalario. Así mismo se ha encontrado que dentro del análisis de las conclusiones mediante sus dos diseños, en primera instancia los descriptivos han demostrado la existencia de una prevalencia del SB a partir de $4.1 \%$ al $100 \%$, de igual modo en los dos únicos casos registrados como correlacionales han presentado coherencia con los objetivos de encontrar relación con aspectos de sexo, edad y otros, de este modo se asocia con los resultados alcanzados por Toala (2019) quien demuestra una prevalencia promedio de 57\% con una grave afección a la salud mental de los residentes; en tanto Loya-Murguía et al. (2018) evidencia un Síndrome de Agotamiento con un intervalo de $2.1 \%$ a $76 \%$, entonces esta representa un nivel de vulnerabilidad al estado mental.

Si bien los resultados del estudio han presentado consistencia tanto con los antecedentes evaluados previamente, estos han presentado algunas limitaciones, como es el caso de la data, donde sólo se consideró dos idiomas, español y portugués, porque los países latinoamericanos hacen uso del mismo, y con respecto al 
portugués con justa razón, siendo la sintomatología más evaluada, de igual manera en el proceso de depuración se ha presentado problemas en Redalyc donde se tuvo que efectuar mayores criterios de exclusión y obtener un número prudente bajo los criterios científicos, otra de las limitaciones presentadas es que el tipo de estudio no ha permitido obtener conclusiones más específicas como lo podría hacer un metaanálisis en tanto la muestra solo se limitó a personal médico, además se pudo haber establecido otros características a fin de ser sistematizadas, luego del contraste de la información, el estudio busca ahondar conocimientos más significativos a la prevalencia de burnout en el personal médico, que dado la realidad actual es necesario que oriente un estudio de metaanálisis para la sistematización de burnout en personal médico en primera línea que han venido luchando contra la Covid19.

\section{CONCLUSIONES}

De acuerdo con la revisión efectuada se ha evidenciado que la mayoría de los artículos proceden de Brasil 14, seguido por Perú con 8 artículos, la totalidad de los estudios corresponden a un tipo aplicado, siendo 41 descriptivos trasversales, 2 de diseño correlacional, que analizaron el SB y sus componentes, la muestra fue en su totalidad personal médico llegando a 11693 sujetos, entre las cuales se encontraron médicos, enfermeros, residentes pediátricos, intensivistas y médicos especialistas.

Los objetivos con mayor descripción fueron los de determinar la prevalencia del síndrome de burnout en el personal médico (16) siendo estos pertenecientes al tipo de estudio aplicada descriptivo transversal, de igual manera se evidenció que el 94\% (41 artículos) han hecho uso del Inventario de Burnout de Maslach para su evaluación, finalmente, se evidencia una alta prevalencia del síndrome de burnout al igual que en sus componentes, partiendo desde $4.1 \%$ hasta $100 \%$ del personal médico evaluado, en cuanto a sus componentes ha tenido una prevalencia mayor al $50 \%$ en la realización personal.

\section{Rol de los autores / Authors roles:}

CTM: realizó la sistematización de los artículos

ENMG: realizó antecedentes de estudio

EMRC: realizó el apartado metodológico de la revisión

EBSG: efectuó las correcciones a nivel teórico del estudio

\section{Conflicto de intereses / Competing interests:}

Los autores no incurren en conflictos de intereses 


\section{REFERENCIAS}

Acosta, J., Morales, L., Álvarez, G. \& Pino, Y. (2019). Síndrome de desgaste profesional en médicos del Hospital Docente Pediátrico Cerro. Revista Habanera de Ciencias Médicas, 18(2), 336-345. http://www.redalyc.org/articulo.oa?id=180460596014

Aguirre, A.M., \& Quijano, A.M. (2015). Síndrome por quemarse en el trabajo y variables familiares y laborales de los médicos generales de Bogotá. Una estrategia de calidad laboral. Revista colombiana de Psiquiatría, 44(4), 198-205. http://dx.doi. Org/10.1016/j.rcp.2015.05.017

Alvares, M.E.M., Thomaz, E.B.A.F., Lamy, Z.C., Nina, R.V.A.H., Pereira, M.U.L., \& Garcia, J.B.S. (2020). Síndrome de burnout entre profissionais de saúde nas unidades de terapia intensiva: um estudo transversal com base populacional. Revista Brasileira de Terapia Intensiva, 32(2), 251-260. https://doi.org/10.5935/0103-507x.20200036

Álvarez, J., Cobo, N., Parra, L., Gómez, L. y Acosta, M. (2019). Prevalencia del síndrome de Burnout en médicos iberoamericanos entre 2012 y 2018: una revisión sistemática. Diálogo de saberes, 50(1), 39-60. https://doi.org/10.18041/0124-0021/dialogos.50.2019.5551

Arayago, R., González, Á., Limongi, M. \& Guevara, H. (2016). Síndrome de Burnout en residentes y especialistas de anestesiología. Salus, 20(1), 13-21. http://www.redalyc. org/articulo.oa?id=375946213004

Athié, C., Cardiel, L.E.; Camacho, J., Mucientes, V.M., Terronez, A.M., Cabrera, N.A., Cueto, H.D., García, G.S., J, A.D., Sánchez, D., Valdés, A.L., \& Sainos, C.A. Burnout en médicos internos de pregrado del Hospital General de México Dr. Eduardo Liceaga. Investigación en Educación Médica, 5(18), 102-107. http://dx.doi.org/10.1016/j. riem.2016.01.020

Ato, M., \& Vallejo, G. (2015). Diseño de investigación en Psicología. Pirámide

Ato, M., López, J.J., \& Benavente, A. (2013). Un sistema de clasificación de los diseños de investigación en psicología. Anales de psicología, 29(3). 1038-1059. http://dx.doi. org/10.6018/analesps.29.3.178511

Baldonedo-Mosteiro, M., Almeida, M.C., Baptista, P.C.P., Sánchez-Zaballos, M., Rodriguez-Diaz, F.J., \& Mosteiro-Diaz, M.P. (2019). Síndrome burnout en trabajadores de enfermería brasileños y españoles. Revista Latino-Americana de Enfermagem, 27, e3192. https://doi.org/10.1590/1518-8345.2818.3192

Bedoya, E.A. (2017). Prevalencia del síndrome de burnout en trabajadores de un hospital público colombiano. Medisan, 21(11). https://www.medigraphic.com/pdfs/medisan/ mds-2017/mds1711e.pdf

Bologna, E. (2013). Estadística para psicología y educación. ( $3^{\mathrm{a}}$ ed.). Editorial Brujas

Bond, M.M., Oliveira, M.S., Bressan, B.J., Bond, M.M.., Silva, A.L., \& Merlo, Á.R. (2018). Prevalência de Burnout entre Médicos Residentes de um Hospital Universitário. Revista Brasileira de Educação Médica, 42(3), 97-107. https://doi.org/10.1590/198152712015v42n3rb20170034.r3 
Bresó-Esteves, E., Pedraza-Álvarez, L., \& Pérez-Correa, K. (2019). Síndrome de burnout y ansiedad en médicos de la ciudad de Santa Marta. Duazary, 16(2), 259-269. https:// doi.org/10.21676/2389783X.2958

Carballo, R., Román, J.J., Rosado, Y.R., \& Rosado, Y.I. (2014). Estrés y síndrome de burnout en estomatólogos. Revista Cubana de Salud y Trabajo, 15(1), 42-49. https:// www.medigraphic.com/pdfs/revcubsaltra/cst-2014/cst141h.pdf

Castillo, G., Rosas, L., Cajias, P., \& Escobar, K. (2019). Identificación del Síndrome de Burnout en personal médico del área de emergencias en un hospital de segundo nivel en Machala-Ecuador. Ciencia y salud virtual, 11(2), 79-89. https://dx.doi. org/10.22519/21455333.1282

Castro, C.S., Timenetsky, K.T., Katz, M., Corrêa, T.D., Felício, A.C., Moriyama, T., Kernkraut, A.M., Ferraz, L.J.R., \& Serpa, A. (2020). Síndrome de burnout e engajamento em profissionais de saúde: um estudo transversal. Revista Brasileira de Terapia Intensiva, 32(3), 381-390. https://doi.org/10.5935/0103-507x.20200066

Chavarría, R.A., Colunga, F.J., Loria, J., \& Peláez, K. (2017). Síndrome de burnout en médicos docentes de un hospital de 2. ${ }^{\circ}$ nivel en México. Educación Médica, 18(4), 254-261. https://doi.org/10.1016/j.edumed.2016.09.001

Cherniss, C. (1993). Role of professional self-efficacy in the etiology and amelioration of burnout. APA Psyclnfo, 135-149. https://psycnet.apa.org/record/1993-97794-008

Chlquillo-Vega, V., Lama-Valdivia, J.E., \& De la Cruz-Vargas, J.A. (2019). Síndrome de burnout en médicos asistentes del Hospital Nacional Hipólito Unanue de Lima - Perú, 2018. Revista de Neuro-Psiquiatría, 82(3). https://doi.org/10.20453/rnp.v82i3.3570

CIE-11 (2020). CIE-11 para estadisticas de mortalidad y morbilidad. https://icd.who.int/ browse11/1-m/es\#/http://id.who.int/icd/entity/1109546957

Cotrina-Onofre, Y., \& Panez-Mateo, L. (2019). Síndrome de Burnout en médicos de tres hospitales de Huánuco. Revista Peruana de Investigación en salud, 3(3), 127-132. https://orcid.org/0000-0003-2415-4541

Da Silva, H., Souza, H., Mesquita, G.V., Viera, J.L., \& Xavier, F.C. (2017). Prevalencia del síndrome de burnout en intensivistas pediátricos, Revista Interdisciplinaria, 10(3), 39-47. https://dialnet.unirioja.es/servlet/articulo?codigo $=6771924$

De Arco, L. y Castillo, J. A. (2020). Síndrome de Burnout en época de pandemia: caso colombiano. Interconectando Saberes, 10(5), 115-123. https://doi.org/10.25009/is.v0i10.2675

Del Cid, A., Méndez, R., \& Sandoval, F. (2007). Investigación: Fundamentos y metodología. Pearson Educación. https://n9.cl/xspa

Díaz, F. y Gómez, I. (2016). La investigación sobre el síndrome de burnout en Latinoamérica entre 2000 y el 2010. Psicología desde el Caribe, 33(1), 113-131. http://dx.doi. org/10.14482/psdc.33.1.8065

Drumond, A.J., Borborema, C., Ferreira, L Fagundes, M., \& de Pinho, L. (2018). Síndrome de Burnout em Médicos de Estratégia Saúde da Família de Montes Claros, MG, e Fatores Associados. Revista Brasileira de Medicina de Familia e Comunidade, 13(40), 1-15. http://dx.doi.org/10.5712/rbmfc13(40)1751 
Dutra, H.S., Gomes, P.A.L., García, R.N., Oliveira, H.C., Freitas S.C., \& Guirardello, E.B. (2019). Burnout entre profissionais de enfermagem em hospitais no Brasil. Revista Cuidarte, 10(1). http://dx.doi.org/10.15649/cuidarte.v10i1.585

Edelwich, J. \& Brodsky, A. (1980): Burnout: Stages of Disillusionment in the Helping Professions. Human Science Press. https://doi.org/10.1177/019263658106544520

Foronda, D., Ciro, D. y Berrío, N. (2018). Síndrome de burnout en personal de la Salud latinoamericano. Revista de Psicología Universidad de Antioquia, 10(2), 157-181. http://dx.doi.org/10.17533/udea.rp.v10n2a07

Freille, D.G., Gazzoni, F., Claros, I.A., Curti, F. S., \& Álvarez, B.A. (2020). Síndrome de Burnout en médicos residentes de la Clínica Universitaria Reina Fabiola. Revista Methodo, 5(3), 88-92. https://doi.org/10.22529/me.2020.5(3)3

García-Moran, M. y Gil-Lacruz, M. (2016). El estrés en el ámbito de los profesionales de la salud. http://bvs.minsa.gob.pe/local/MINSA/4131.pdf

Gonzales, J., Arbeláez, J.S., Lopera, N.M., \& Valderrama, K.J. (2018). Prevalencia del síndrome de burnout en parte del personal asistencial de una clínica oncológica de la ciudad de Armenia (Quindío, Colombia). Archivos de Medicina, 18(1). https://doi. org/10.30554/archmed.18.1.2156.2018

Hernández, R., Fernández, C. y Baptista, P. (2014). Metodología de la investigación. (6 ${ }^{\mathrm{a}}$ ed.). Mc Graw Hill Education

Hernández-García, J. (2018). Burnout en médicos de un Hospital del sector público en el Estado de Hidalgo. Agricultura, sociedad y desarrollo, 15, 161-172. https://doi. org/10.22231/asyd.v15i2.799

Hoppen, C.M., Kissmann, N., Chinelato, J.R., Coelho, V.P., Wenczenovicz, C., Nunes, F.C., \& Friedman, G. (2017). Alta prevalência de síndrome de burnout em médicos intensivistas da cidade de Porto Alegre. Revista Brasileira de Terapia Intensiva, 29(1), 115-120. https://doi.org/10.5935/0103-507x.20170017

Huarcaya-Victoria, J., \& Calle-Gonzáles, R. (2020). Influencia del síndrome de burnout y características sociodemográficas en los niveles de depresión de médicos residentes de un hospital general. Educación médica. https://doi.org/10.1016/j.edumed.2020.01.006

Jácome, S.J., Villaquiran-Hurtado, A., García, C.P., \& Duque, I.L. (2019). Prevalencia del síndrome de Burnout en residentes de especialidades médicas. Revista Cuidarte, 10(1), 1-12. http://dx.doi.org/10.15649/cuidarte.v10i1.543

Juárez-García, A., Idrovo, A.J., Camacho-Ávila, A., \& Placencia-Reyes, O. (2014). Síndrome de burnout en población mexicana: Una revisión sistemática, Salud Mental, 37, 159-176. https://www.redalyc.org/pdf/582/58231307010.pdf

Lima, A.S., Farah, B.F., \& Bustamante-Teixeira, M.T. (2018). Análise da prevalência da síndrome de burnout em profissionais da atenção primária em saúde. Trabalho, Educação e Saúde, 16(1), 283-304. https://doi.org/10.1590/1981-7746-sol00099

Losada, H., Curitol, S., Astudillo, P., Shaneeberger, P., \& Coronado, F. (2018). Prevalencia de Síndrome de Burnout en un Centro de Cirugía Académico-Asistencial 
Público en Chile. Rev Chil Cir, 70(2), 117-126. http://dx.doi.org/10.4067/s071840262018000200117

Loya-Murguía, K., Valdez-Ramírez, J., Bacardí-Gascón, M. y Jiménez-Cruz, A. (2018). El síndrome de agotamiento en el sector salud de Latinoamérica: revisión sistemática. Journaal of negative no positive results, 3(1), 40-48. https://www.jonnpr.com/ pdf/2060.pdf

Lugo, J.F. (2019). Síndrome de Burnout: factores asociados en médicos de un hospital tipo III. Revista digital de Posgrado, 8(1). http://portal.amelica.org/ameli/jatsRepo/101/101599005/html/

Luz, L.M., Torres, R.R.B., Sarmento, K.M.V.Q., Sales, J.M. Farias, K.N., \& Marques, MB. (2017). Burnout Syndrome in urgency mobile service professionals. RevFund Care Online, 9(1), 238-246. http://dx.doi.org/10.9789/2175-5361.2017.v9i1.238-246

Marques, G.L.C., Carvalho, F.L, Fortes, S., Miranda, H.R. \& Alves, G.S. (2018). Síndrome de burnout entre médicos plantonistas de unidades de terapia intensiva. Jornal Brasileiro de Psiquiatria, 67 (3), 186-193. https://doi.org/10.1590/0047-2085000000202

Martínez, P. (2019). Una revisión bibliográfica: El síndrome de Burnout. Ucronos. 12(1). https://revistamedica.com/sindrome-de-burnout/

Martins, N., de Moura, E., de Alburquerque, D., Silva, N., \& Muniz, M.T. (2019). Síndrome de burnout em médicos residentes. Revista Médica UFC, 59(3), 20-23. http:// dx.doi.org/10.20513/2447-6595.2019v59n3p20-23

Maslach, C., Schaufeli, W., \& Leiter, M. (2001). Job Burnout. Annual Review of Psychology, 52, 397-422. https://doi.org/10.1146/annurev.psych.52.1.397

Maticorena-Quevedo, J., Beas, R., Anduaga-Beramendi, A. y Mayta-Tristán, P. (2016). Prevalencia del síndrome de burnout en médicos y enfermeras del Perú, ENSUSALUD 2014. Revista Peruana de Medicina Experimental y Salud Pública, 33(2). https://rpmesp.ins.gob.pe/index.php/rpmesp/article/view/2170/2233

Maticorena-Quevedo, J., Beas, R., Anduaga-Beramendi, A., Mayta-Tristan, P. (2016). Prevalencia del síndrome de burnout en médicos y enfermeras del Perú, Ensusalud 2014. Rev Peru Med Exp Salud Publica, 33(2), 241-247. https://doi.org/10.17843/rpmesp.2016.332.2170

Medina, M.L., Medina, M.G., Gauna, N.T., Molfino, L., \& Merino, L.A. (2017). Prevalencia del síndrome de burnout en residentes de Pediatría de un hospital Investigación. Educación Médica, 6(23), 160-168. http://dx.doi.org/10.1016/j.riem.2017.03.004

Miguel, S. (2011). Revistas y producción científica de América Latina y el Caribe: su visibilidad en Scielo, Redalyc y Scopus. Revista Interamericana de Bibliotecología, 34(2), 187-199. http://www.scielo.org.co/pdf/rib/v34n2/v34n2a6.pdf

Ministerio de la Mujer y Poblaciones Vulnerables-MIMP (2017). Conociendo el síndrome de agotamiento profesional. http://bvs.minsa.gob.pe/local/MINSA/4026.pdf

Ministerio de Salud-Minsa (2020). Cuidado de la salud mental del personal de la salud en el contexto del Covid-19. http://bvs.minsa.gob.pe/local/MINSA/5000.pdf 
Minsalud (2015). Prevenir el cansancio en el personal de salud. https://n9.cl/o67lf

Montero, I., \& León, O. (2002). Clasificación y descripción de las metodologías de investigación en Psicología. Revista Internacional de Psicología Clínica y de la Salud, 2(3), 503-508.http://www.aepc.es/ijchp/articulos_pdf/ijchp-53.pdf

Mugnani, R. (2018). Producción e impacto de la investigación brasileña: confrontando contextos internacional y nacional, Scielo en Perspectiva. https://n9.cl/6hmi9

Muñoz, A., Arias, W.L., \& Caycho-Rodríguez, T. (2019). Síndrome de burnout en médicos de la ciudad de Arequipa (Perú). Revista Chilena de Neuro-Psiquiatría, 57(2), 139148. http://dx.doi.org/10.4067/S0717-92272019000200139

Muñoz, A.I., \& Velásquez, M.S. (2016). Síndrome de quemarse por el trabajo en profesionales de enfermería, Bogotá, Colombia. Rev. Fac. Nac. Salud Pública, 34(2), 202211. https://doi.org/10.17533/udea.rfnsp.v34n2a09

Muñoz, S., Ordonez, J.N., Solarte, M.N., Valverde, Y.C., Villareal, S., \& Zemanate, M.L (2018). Síndrome de Burnout en enfermeros del Hospital Universitario San José. Popayán. Revista Médica de Risaralda, 24(1), 34-37. http://www.scielo.org.co/pdf/rmri/ v24n1/v24n1a06.pdf

Ñaupas, H., Valdivia, M., Palacios, J., y Romero, H. (2018). Metodología de la investigación cuantitativa-cualitativa y redacción de la tesis. ( $5^{\mathrm{a}}$ ed.) Ediciones de la U. https:/corladancash.com/wp-content/uploads/2020/01/Metodologia-de-la-inv-cuanti-y-cuali-Humberto-Naupas-Paitan.pdf

Oliveira, B., Rodrigues, T., Figueiredo, F., Nascimiento, M., Cavalcanti, R., \& Oliveira, M. (2015). Prevalência da síndrome de burnout em médicos de uma instituição hospitalar federal de ensino. Journal of Research Fundamental Care Online, 7, 39-48. https://www.redalyc.org/pdf/5057/505750949004.pdf

Organización Mundial de la Salud -OMS (2019). El agotamiento, un fenómeno ocupacional: Clasificación Internacional de Enfermedades. https://www.who.int/mental_ health/evidence/burn-out/en/

Ortega, G., Pio, G., Cardemil, F., \& Sade, C. (2015). Presencia de síndrome de Burnout en una muestra de residentes y otorrinolaringólogos de Chile. Revista de Otorrinolaringología y Cirugía de Cabeza y Cuello, 75, 227-231. http://dx.doi.org/10.4067/ S0718-48162015000300005

Otzen, T., \& Manterola, C. (2017). Técnicas de muestreo sobre una población a estudio. International Journal of Morphology, 35(1), 227 - 232. https://scielo.conicyt.cl/pdf/ ijmorphol/v35n1/art37.pdf

Price, D., \& Murphy, P. (1984). Staff Burnout in the Perspective of Grief Theory. Death Education, 8(1), 47-58. http://dx.doi.org/10.1080/07481188408251381

Ramírez, M. (2017). Prevalencia del síndrome de burnout y la asociación con variables sociodemográficas y laborales en una provincia de Ecuador. International Journal of Developmental and Educational Psychology, 4(1), 240-251. https://www.redalyc.org/ pdf/3498/349853537025.pdf 
Ramírez, M., \& Ontaneda, M. (2019). Prevalencia del síndrome de burnout y su relación con el afrontamiento en profesionales de la salud de la zona 7. European Journal of Health Research, 5(2), 75-84. https://dialnet.unirioja.es/servlet/articulo?codigo=7562674

Ramírez, R., Meneses-Echavez, J.F., \& Floréz-López, M.E. (2013). Una propuesta metodológica para la conducción de revisiones sistemáticas de la literatura en la investigación biomédica. CES Movimiento y Salud, 1, 61-73. http://132.248.161.133:8080/jspui/ handle/123456789/5467

Rojas, M. (2015). Tipos de Investigación científica: Una simplificación de la complicada incoherente nomenclatura y clasificación, Redvet, 16(1), 1-14. https://www.redalyc. org/pdf/636/63638739004.pdf

Saborio, L. y Hidalgo, L. (2015). Síndrome de Burnout. Medicina Legal de Costa Rica, 32(1). https://www.scielo.sa.cr/pdf/mlcr/v32n1/art14v32n1.pdf

Sánchez, F.A. (2019). Fundamentos Epistémicos de la Investigación Cualitativa y Cuantitativa: Consensos y Disensos. Revista Digital de Investigación en Docencia Universitaria, 13(1), 102-122. https://doi.org/10.19083/ridu.2019.644

Sarmiento, G.S. (2019). Burnout en el servicio de emergencia de un hospital. Horiz Med, 19(1), 67-72. http://dx.doi.org/10.24265/horizmed.2019.v19n1.11

Seguel, F.S., \& Valenzuela, S. (2016). Síndrome de burnout en trabajadores de enfermería de dos hospitales del sur de Chile. Avances en enfermería, 34(1), 39-47. http://dx.doi. org/10.15446/av.enferm.v34n1.41599

Serrano, M.A., Rangel, A., Vidal, C.R., Ureña, Y.C., Anillo, H., \& Angulo, G. (2017). Burnout: Síndrome silencioso que afecta el desempeño laboral de los docentes universitarios. Revista Espacios, 38(55). https://www.revistaespacios.com/a17v38n55/ a17v38n55p28.pdf

Silva, R., \& Drogas, F. (2001). Ética y trasgresión. Montevido Piscolibros, 57-77.

Solís-Cóndor, A., Tantalean-del Águila, M., Burgos-Aliaga, R., \& Chambi-Torres, J. (2017). Agotamiento profesional: prevalencia y factores asociados en médicos y enfermeras en siete regiones del Perú. An Fac med., 78(3), 270-276. ttp://dx.doi. org/10.15381/ana- les.v78i3.13757

Solís-Cóndor, R., Tantalean-del Águila, M., Burgos-Aliaga, R. y Chambi-Torres, J. (2017). Agotamiento profesional: prevalencia y factores asociados en médicos y enfermeras en siete regiones del Perú. Anales de la Facultad de Medicina, 78(3), 270-276. https:// dx.doi.org/10.15381/anales.v78i3.13757

Supo, J. (2014). Como elegir una muestra: técnicas para seleccionar una muestra representativa. Bioestadístico

Tabares-Díaz, Y.A., Martínez-Daza, V.A., \& Matabanchoy-Tulcán, S.M. (2020). Síndrome de Burnout en docentes de Latinoamérica: Una revisión sistemática. Universidad y Salud, 22(3), 265-279. https://dx.doi.org/10.22267/rus.202203.199

Terrones-Rodríguez, J.F., Cisneros-Pérez, V., \& Arreola-Rocha, J.J. (2016). Síndrome de burnout en médicos residentes del Hospital General de Durango, México. Revista 
Médica del Instituto Mexicano del Seguro Social, 54(2), 242-248. http://www.redalyc.org/articulo.oa?id=457745149016

Tironi, M. O., Teles, J. M., Barros, D., Vieira, D. F., Silva Filho, C., Martins Júnior, D. F., Matos, M. A., \& Nascimento Sobrinho, C. L. (2016). Prevalência de síndrome de burnout em médicos intensivistas de cinco capitais brasileiras. Revista Brasileira de Terapia Intensiva, 28(3), 270-277. https://doi.org/10.5935/0103-507X.20160053

Toala, J. (2019). Síndrome de Burnout en Médicos Residentes. Revista San Gregorio, (33), 102-113. https://dx.doi.org/10.36097/rsan.vli33.966

Torrecillas, F., Gómez, P. y Pérez, C. (2020). Impacto emocional expresado como síndrome de burnout en los profesionales de enfermería, a partir de las situaciones originadas por el coronavirus COVID-19. Revista Electrónica de PortalesMedicos.com, 15(10), 383. https://n9.cl/76jf

Torres, A.D., \& Alcaraz, I.A. (2020). Síndrome de Burnout en médicos residentes de un Hospital público de la ciudad de Areguá en el año 2020. Medicina Clínica y social, 4(3), 98-103. https://medicinaclinicaysocial.org/index.php/MCS/article/view/151

Urra, E., \& Barria, R.M. (2010). La revisión sistémica y su relación con la práctica basada en la evidencia en salud. Rev. Latino-Am. Enfermagem, 18(4). https://www.scielo.br/ pdf/rlae/v18n4/es_23.pdf

Vidotti, V., Martins, J.T., Galdino, M.J.Q., Ribeiro, R.P., \& Robazzi, M.L. (2019). Síndrome de burnout, estrés laboral y calidad de vida en trabajadores de enfermería. Enfermería Global, 18(55), 344-376. https://dx.doi.org/10.6018/eglobal.18.3.325961

Vinueza, A., Aldaz, N., Mera, C., Pino, D., Tapia, E. y Vinueza, M. (2020). Síndrome de Burnout en médicos/as y enfermeros/as ecuatorianos durante la pandemia de COVID-19. http://dx.doi.org/10.1590/SciELOPreprints.708

Vivanco, M.E., Sánchez, C.D., Maldonado, R.P., \& Enrique, E.R.N. (2018). Síndrome de burnout en profesionales de la salud en instituciones públicas y privadas: un análisis en la provincia de Loja, Ecuador. Revista Boletin Redipe, 7(11), 179-194. https://revista.redipe.org/index.php/1/article/view/622/579

Zanatta, A. B., \& Lucca, S.R. (2015). Prevalência da síndrome de burnout em profissionais da saúde de um hospital oncohematológico infantil. Revista da Escola de Enfermagem da USP, 49(2), 253-258. https://dx.doi.org/10.1590/S0080-623420150000200010 
